\title{
Potencial para maricultura da carragenófita Hypnea musciformis (Wulfen) J.V. Lamour. (Gigartinales - Rhodophyta) na Ilha da Marambaia, Baía de Sepetiba, RJ, Brasil
}

\author{
Renata Perpetuo Reis ${ }^{1,3}$, Aline Queiroz Caldeira ${ }^{1}$, Ana Paula dos Santos Miranda ${ }^{1}$ e \\ Maria Beatriz Barros-Barreto ${ }^{2}$
}

Recebido em 7/11/2005. Aceito em 17/04/2006

\begin{abstract}
RESUMO - (Potencial para maricultura da carragenófita Hypnea musciformis (Wulfen) J.V. Lamour. (Gigartinales - Rhodophyta) na Ilha da Marambaia, Baía de Sepetiba, RJ, Brasil). A falta da prática de cultivo comercial de macroalgas no Brasil junto com a crescente demanda do mercado nacional de ficocolóides é suprida pela importação de algas e colheita em bancos naturais, principalmente na costa nordestina. Na busca de um cultivo em pequena escala para uso das comunidades litorâneas foi implantado um cultivo experimental com H. musciformis, na Ilha da Marambaia, RJ (43059'35" W e 2304'00" S). Ao todo foram testadas nove técnicas de fixação da alga nas unidades experimentais de maricultura (UEM). Nos experimentos realizados entre a primavera de 2003 e o outono de 2004, as taxas de crescimento geralmente foram negativas. Entre os invernos de 2004 e 2005, foram na maioria positivas. O fitobentos próximo à UEM foi analisado para verificar o crescimento dessa espécie na assembléia e no cultivo. Observou-se maior percentual de anfípodas e de matéria orgânica nas UEM e a presença de carposporófitos em épocas de baixa salinidade em ambos os locais. Atribuiu-se o baixo rendimento da taxa de crescimento aos fatores ambientais como: ressacas, aderência de matéria orgânica nas algas, herbivoria e danos as UEM por ação antrópica. Constatou-se que o tratamento fixando a muda com abraçadeira foi o de mais fácil manuseio e baixo custo e que somente com a conscientização da comunidade litorânea e remuneração salarial para esta atividade será possível exercer o cultivo de H. musciformis com sucesso.
\end{abstract}

Palavras-chave: Maricultura, macroalgas, H. musciformis, carragenana, Ilha da Marambaia

\begin{abstract}
Mariculture potential of carragenophyta Hypnea musciformis (Wulfen) J.V. Lamour. (Gigartinales - Rhodophyta) on Marambaia Island, Baía de Sepetiba, Rio de Janeiro State, Brazil). Lack of practice in commercial cultivation of macroalgae in Brazil allied with an increased demand for phycocolloids in the national market is met by importing algae and cropping from natural beds, especially on the Northeastern coast. Seeking to establish small scale cultivation for the use of coastal communities, experimental cultivation was implanted with $H$. musciformis on Marambaia Island, Rio de Janeiro (4359'35" W - 2304'00" S). Nine different techniques for fixation of algae to ropes were tested in the experimental mariculture units (EMU). Experiments carried out from spring 2003 to fall 2004 yielded generally negative growth rates while experiments conducted from winter 2004 to winter 2005 yielded positive growth rates. Phytobenthos near the EMU were analyzed to verify the growth of this species in the assemblage and in cultivation. A higher percentage of amphipods and of organic matter was observed in the EMU as well as the presence of carposporophytes during low salinity periods at both sites. This low yield was attributed to environmental factors such as rough seas, adherence of organic matter to the algae, herbivory and EMU damages caused by anthropic activities. Fixing the algae with a clamp was the easiest and cheapest technique. Successful cultivation of $H$. musciformis can only be achieved through consciousness raising in the coastal community and payment for this activity.
\end{abstract}

Key words: Mariculture, macroalgae, H. musciformis, carrageenan, Marambaia Island

\section{Introdução}

A falta da prática de cultivo comercial de macroalgas no Brasil (Oliveira 1998; Marinho-Soriano et al. 2002), aliada à crescente demanda do mercado nacional de ficocolóides é suprida pela importação de macroalgas e pela colheita em bancos naturais ou de algas arribadas (Oliveira 1998; Furtado 1999).

\footnotetext{
1 Instituto de Pesquisas Jardim Botânico do Rio de Janeiro, Ministério do Meio Ambiente, Programa Zona Costeira (JBRJ), Rua Pacheco Leão 915, 22460-030 Rio de Janeiro, RJ, Brasil

2 Universidade Federal Rural do Rio de Janeiro, Instituto de Biologia, Departamento de Botânica, Antiga Rio-São Paulo, Km 47, $23851-970$ Seropédica, RJ, Brasil (beatrizb@ufrrj.br)

3 Autor para correspondência: rreis@jbrj.gov.br
} 
Atualmente, comunidades litorâneas nordestinas (Rio Grande do Norte, Ceará e Paraíba) vêm sendo orientadas para o uso racional desse recurso marinho, substituindo a atividade extrativista pela sustentável, a maricultura (Carvalho Filho 2004; Miranda et al. 2004). Três empresas nacionais processam agaranas e carragenanas no Brasil: a Griffith do Brasil, com sede em Mogi das Cruzes (SP), que importa algas das Filipinas; a Agar Gel, localizada em João Pessoa (PB), que produz carragenana de $H$. musciformis (Wulfen) J.V. Lamour. e agar de espécies de Gracilaria Grev. provenientes da costa nordestina e de Gracilaria chilensis C.J. Bird, McLachlan \& E.C. Oliveira importada do Chile (Furtado 1999; Carvalho Filho 2004) e a empresa Sete Ondas Biomar, inaugurada em 2005, no Estado do Rio de Janeiro, para processar carragenana semi refinada de Kappaphycus alvarezi (Doty) Doty, cultivada na Baía da Marambaia por esta empresa (observação pessoal). Ressalta-se a possibilidade de danos ambientais ocasionados pela introdução de espécies exóticas, como acúmulo de biomassa sobre recifes causando a morte de corais por sombreamento, como também a sua introdução com sucesso em locais com temperatura baixas (menores que $20^{\circ} \mathrm{C}$ ) e água turva (Paula et al. 1998).

$\mathrm{O}$ custo da alga seca depende da qualidade, limpeza e secagem da mesma (Accioly 2005). A alga chilena, por exemplo, devido à sua melhor qualidade, é quase quatro vezes mais cara do que a brasileira (Carvalho Filho 2004). Quando o tipo de ficocolóide está relacionado ao estágio do ciclo reprodutivo da espécie, as algas provenientes da maricultura são mais caras, devido à possibilidade de selecionar o tipo de ficocolóide que será extraído (Romo, comunicação pessoal).

O conhecimento sobre as técnicas ideais para maricultura é obtido através de estudos experimentais relacionados aos aspectos ambientais e biológicos (Santelices 1999; Marinho-Soriano 2005). Desse modo, ampliar o conhecimento sobre a biologia de espécies brasileiras com potencial para maricultura e sobre os métodos apropriados de cultivo massivo torna-se urgente para a exploração racional de bancos naturais destas espécies assim como para a implementação de cultivos com espécies nativas (Accioly 2005; MarinhoSoriano 2005). Em estudo prévio sobre maricultura de algas vermelhas de interesse comercial na Praia do Kutuca, na Ilha da Marambaia, município de Mangaratiba, Estado do Rio de Janeiro (Reis et al. 2005), H. musciformis apresentou a maior taxa de crescimento, quando comparada com duas espécies de agarófitas (Gracilaria caudata J. Agardh e G. cervicornis (Turner) J. Agardh), tanto em cultivos in situ quanto in vitro. A partir desta observação, foi avaliado o potencial de $H$. musciformis para maricultura neste local através de diferentes técnicas de cultivo.

\section{Material e métodos}

Mudas de Hypnea musciformis foram coletadas na Praia do Kutuca, na Ilha da Marambaia, município de Mangaratiba, RJ (43059'35" W e 2304'00" S) e fixadas em quatro unidades experimentais de maricultura (UEM), com sub-amostras distanciadas entre si cerca de 2 metros. Cada UEM foi confeccionada com um par de poitas de cimento (ou tijolos de concreto), com uma ponta amarrada a uma corda de nylon $(6 \mathrm{~mm})$ e a outra a um flutuador. Dois flutuadores uniram as cordas de polietileno de $10 \mathrm{~m}$ de comprimento, usualmente em maricultura este tipo de cultivo é denominado "long-line". O "long line" foi mantido paralelo à superfície da água, por meio de flutuadores, submersos aproximadamente a $50 \mathrm{~cm} \mathrm{e}$ distanciados transversalmente do costão rochoso cerca de10 metros. Inicialmente as UEM foram dispostas em local mais distante da praia. Posteriormente, foram transferidas para um local mais resguardado e próximo à praia.

Ao longo de 21 meses (novembro/2003 a julho/2005) as mudas de H. musciformis foram fixadas nos "long-line" isoladas ou em diferentes conjuntos de tratamentos: $(\mathrm{A})$ = Fixada diretamente ao "long-line", com abraçadeira; $(\mathrm{AC})=$ Fixada com abraçadeira ao cabo de polietileno; $(\mathrm{CPD})=$ Fixada com abraçadeira ao cabo de polietileno desfiado; $(\mathrm{CS} 1)=$ Fixada com uma abraçadeira ao cabo de seda desfiado; $(\mathrm{CS} 2)=$ Fixada com duas abraçadeiras ao cabo de seda desfiado; $(\mathrm{E})$ = Entremeada no cabo de polietileno; $(\mathrm{GC})=$ Fixada com abraçadeira em Gracilaria cervicornis; $(\mathrm{S})$ = Fixada com abraçadeira em Sargassum $\mathrm{sp}$. e $(\mathrm{SN})=$ inserida em saco de nylon com malha de cerca de $1 \times 1 \mathrm{~cm}$. Os pesos das mudas perdidas de cada tratamento foram considerados zero na ocasião do cálculo da média de cada tratamento.

Após cada coleta, os tratamentos foram retirados das UEM e colocados imediatamente em sacos plásticos para evitar a perda de invertebrados. No laboratório, as amostras foram lavadas, separados os organismos e H. musciformis foi triada de acordo com o seu estado reprodutivo. Posteriormente, o material foi seco em estufa a $60{ }^{\circ} \mathrm{C}$, até obtenção de massa 
constante. $\mathrm{O}$ crescimento foi estabelecido pela taxa de crescimento relativo (\% ao dia), através da fórmula $\mathrm{TCR}=100 \times\{\mathrm{Ln}($ peso inicial/peso final $)\} /$ tempo de cultivo (Romo et al. 2001; Marinho-Soriano et al. 2002; Reis et al. 2005).

A proporção de $H$. musciformis, outras algas, invertebrados e silte misturado com massa orgânica foram quantificados através da porcentagem de massa seca em cada tratamento do experimento.

Para verificar a existência de variação sazonal no percentual de carragenana, amostras ao acaso de H. musciformis foram coletadas no costão em diferentes épocas do ano no período estudado. Carragenanas extraídas de algas do costão, coletadas em março/2005, foram comparadas com carragenanas extraídas de algas do cultivo da mesma época. O rendimento da carragenana foi obtido através do cálculo da porcentagem de carragenana extraída de uma amostra (massa seca) de H. musciformis, ou seja, da razão entre a massa seca de carragenana extraída e a massa seca de alga seca utilizada na extração. A extração da carragenana foi realizada através de uma amostra de alga seca em estufa a $60{ }^{\circ} \mathrm{C}$ até atingir massa constante. Esta amostra foi macerada com grau e pistilo até ser reduzida a pó. Foi adicionada água destilada na proporção de 1:100 e esta solução aquecida a $85 \pm 5^{\circ} \mathrm{C}$, com agitação constante durante 1 hora. $\mathrm{O}$ extrato foi filtrado duas vezes em gaze cirúrgica (4 voltas), ainda quente. Ao filtrado, foi adicionado o dobro de seu volume com álcool etílico anidro e filtrado o precipitado. Este precipitado foi seco em estufa a $60{ }^{\circ} \mathrm{C}$ até a obtenção de massa constante. Foram usadas quatro réplicas.

Dados ambientais como salinidade e temperatura da água do mar foram registrados durante os meses de dezembro/2003 a julho/2005, na Praia do Kutuca, Ilha da Marambaia, RJ (Fig. 1). A salinidade foi medida com refratômetro manual (marca American Optical) e a temperatura superficial da água do mar com termômetro manual de mercúrio. Dados sobre a precipitação (Fig. 1), velocidade dos ventos (Fig. 1) e sobre o predomínio de ventos no quadrante norte-leste, relativos à da Base Aérea de Santa Cruz, RJ (2256'S

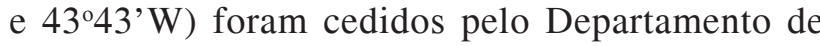
Controle do Espaço Aéreo/Serviços Regionais de Proteção ao Vôo da Aeronáutica (DECEA/SRPV).

A existência de diferenças entre as taxas de crescimento de $H$. musciformis entre os diferentes
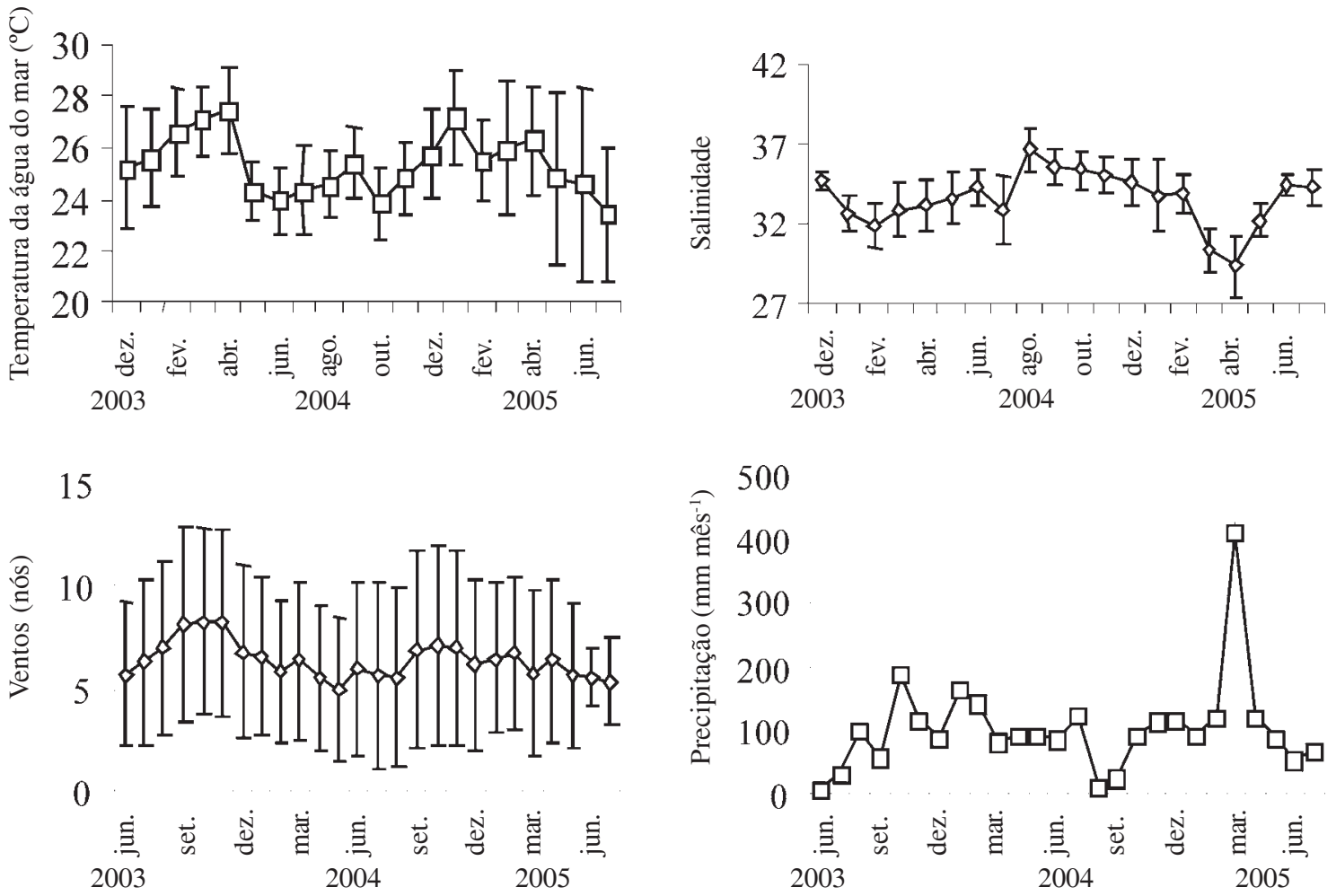

Figura 1. Variação da temperatura e da salinidade da água do mar medidas na Praia do Kutuca, Ilha da Marambaia, RJ e velocidade do vento e precipitação medidos na Base Aérea de Santa Cruz. Pontos significam média e linhas desvio padrão. 
tratamentos de fixação das algas no "long-line" em cada período de cultivo, entre as amostras de carragenana do costão e as do cultivo e entre as carragenana coletadas em diferentes meses no costão foram obtidas pela análise de variância unifatorial (ANOVA). As diferenças entre as médias foram separadas pelo teste de Tukey (Zar 1996). A normalidade das amostras foi verificada pelo teste de Shapiro-Wilk W e a homogeneidade da variância pelo teste de Cochran (Zar 1996). O grau de confiança para todos os testes de significância foi de $95 \%(p=0,05)$. Os dados são apresentados em média \pm desvio padrão.

\section{Resultados e discussão}

As taxas de crescimento de H. musciformis observadas nas diferentes técnicas de fixação de mudas estão apresentadas na Tab. 1. Apesar de não haver diferença entre as técnicas testadas nos experimentos de dezembro/2003, maio/2004, fevereiro/2005 e março/ 2005, constatou-se que o tratamento com a muda fixada com abraçadeira no "long-line" (A) foi o de mais fácil manuseio e baixo custo em relação aos demais, que é o método semelhante ao amarrado com fitilho, sendo este último o menos oneroso. Além da facilidade de manuseio e baixo custo, geralmente os outros tratamentos apresentaram uma tendência a um maior percentual de acúmulo de matéria orgânica e silte (MO) e de invertebrados (Invertebrados), conforme ilustrado na Fig. 2. Os principais invertebrados observados foram anfípodas, ascídias, bivalvos, briozoários e crustáceos e as principais algas identificadas, além de H. musciformis (Outras algas), foram Ulva fasciata Delile, Ulva lactuca Linnaeus, Dictyota cervicornis Kützing, Acanthophora spicifera (M. Vahl) Børgesen, Gracilaria caudata J. Agardh, Gracilaria cervicornis (Turner) J. Agardh e Hypnea spinella (C. Agardh) Kützing.

Acredita-se que a MO que se aderiu nas mudas cultivadas foi transportada do interior da Baía de Sepetiba principalmente em períodos com predomínio de vento de quadrante norte a leste. Em coleta realizada no dia 31 de maio de 2005, a água gradativamente ficou turva com a mudança de vento do quadrante noroeste, que predominaram de 7 às $12 \mathrm{~h}$, para vento do quadrante nordeste, de 12 às $18 \mathrm{~h}$ (dados de ventos do DECEA/

Tabela 1: Experimentos de maricultura com H. musciformis (Wulfen) J.V. Lamour. realizados na Praia do Kutuca, Ilha da Marambaia, RJ. Tipos de tratamentos fixados no "long-line": A = fixada com abraçadeira; AC = fixada com abraçadeira ao cabo de polietileno; CPD = fixada com abraçadeira ao cabo de polietileno desfiado; $\mathrm{CS} 1$ = fixada com uma abraçadeira ao cabo de seda desfiado; CS2 = fixada com duas abraçadeiras ao cabo de seda desfiado; $\mathrm{E}=$ entremeada no cabo de polietileno; $\mathrm{F}$ = fixada com fitilho; $\mathrm{GC}=$ fixada com abraçadeira em Gracilaria cervicornis; $\mathrm{S}=$ fixada com abraçadeira em Sargassum $\mathrm{sp}$.; SN = inserida em saco de nylon com malha de cerca de $1 \times 1 \mathrm{~cm}$.

\begin{tabular}{|c|c|c|c|c|c|c|c|c|}
\hline \multirow[t]{2}{*}{ Coleta } & \multirow{2}{*}{$\begin{array}{l}\text { Tempo } \\
\text { (dias) }\end{array}$} & \multirow[t]{2}{*}{ Tratamento } & \multirow{2}{*}{$\begin{array}{l}\text { Taxas de crescimento } \\
\text { (média } \pm \text { desvio padrão) }\end{array}$} & \multirow[t]{2}{*}{ Diferença } & \multicolumn{4}{|c|}{ ANOVA } \\
\hline & & & & & $\mathrm{n}$ & $\mathrm{F}$ & Valor $\mathrm{p}$ & Resultado \\
\hline Dezembro/2003 & 34 & $\begin{array}{c}\text { E } \\
\text { SN } \\
\text { F }\end{array}$ & $\begin{array}{l}-1,84 \pm 4,32 \\
-5,38 \pm 1,96 \\
-1,35 \pm 2,35\end{array}$ & Ex SN x F & 3 & 1,51 & 0,29 & $\mathrm{E}=\mathrm{SN}=\mathrm{F}$ \\
\hline Janeiro/2003 & 35 & SN & $-8,10 \pm 6,5$ & & & & & \\
\hline Abril/2004 & 20 & SN & $-8,75 \pm 8,01$ & & & & & \\
\hline Maio/2004 & 46 & $\begin{array}{c}\text { AC } \\
\text { CS1 } \\
\text { CS2 } \\
\text { CPD } \\
\text { S } \\
\text { GC }\end{array}$ & $\begin{array}{c}-1,70 \pm 2,96 \\
-0,45 \pm 3,34 \\
-0,38 \pm 0,62 \\
1,07 \pm 0,40 \\
-1,03 \pm 1,79 \\
0\end{array}$ & $\begin{array}{c}\text { AC x CS } 1 \\
\text { CS } 2 \times C P D \times \\
\text { S x GC }\end{array}$ & 3 & 0,67 & 0,63 & $\begin{array}{c}\mathrm{AC}=\mathrm{CS} 1= \\
\mathrm{CS} 2=\mathrm{CPD}=\mathrm{S} \\
=\mathrm{GC}\end{array}$ \\
\hline Agosto/2004 & 42 & CPD & $1,50 \pm 1,31$ & & & & & \\
\hline Fevereiro/2005 & 45 & $\begin{array}{c}\mathrm{A} \\
\mathrm{CPD}\end{array}$ & $\begin{array}{c}0,30 \pm 1,17 \\
0,90 \pm 2,25\end{array}$ & $\mathrm{~A} \times \mathrm{CPD}$ & 4 & 0,88 & 0,38 & $\mathrm{~A}=\mathrm{CPD}$ \\
\hline Março/2005 & 92 & $\begin{array}{c}\mathrm{A} \\
\mathrm{CPD}\end{array}$ & $\begin{array}{c}-1,24 \pm 2,72 \\
1,52 \pm 0,27\end{array}$ & $A \times C P D$ & 3 & 3,01 & 0,16 & $\mathrm{~A}=\mathrm{CPD}$ \\
\hline Abril/2005 & 44 & A & $2,85 \pm 1,10$ & & & & & \\
\hline Julho/2005 & 44 & A & $0,77 \pm 2,52$ & & & & & \\
\hline
\end{tabular}


Dezembro/2003

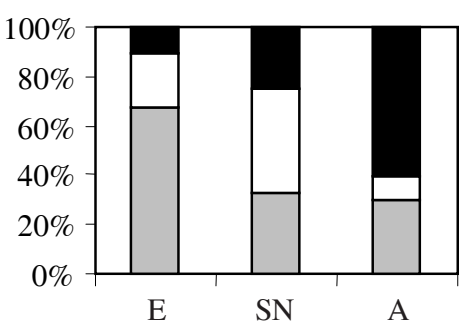

Maio/2004

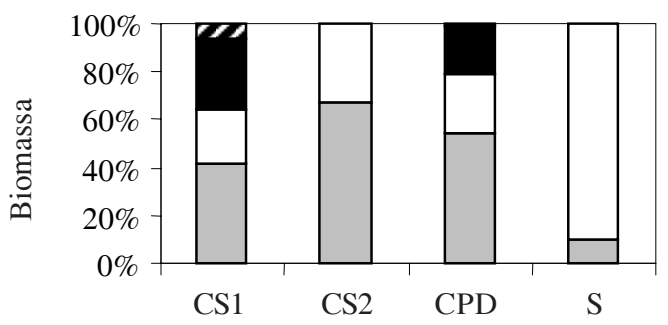

Março/2005

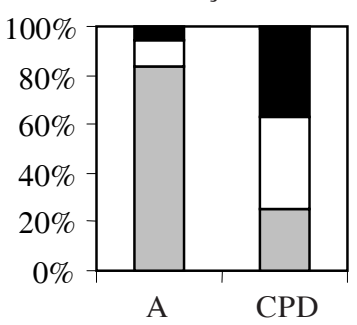

Janeiro/2004

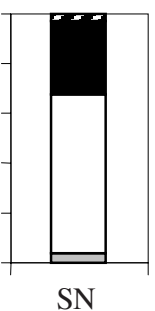

Agosto/2004

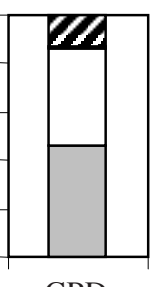

CPD
Abril/2004

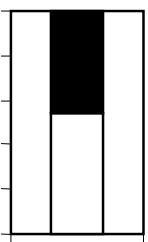

SN

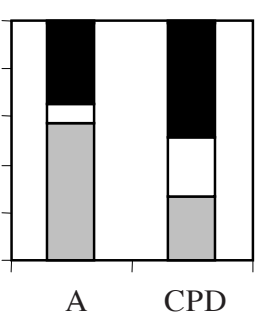

Julho/2005

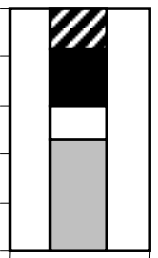

A

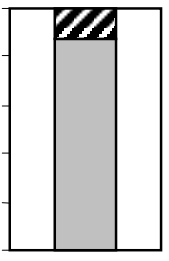

A

Figura 2. Percentual da massa seca de Hypnea musciformis (Wulfen) J.V. Lamour., massa orgânica misturada com silte (MO), anfípodas, ascídias, bivalvos, briozoários e crustáceos (invertebrados) e outras algas (algas), nos tratamentos fixados nos "log-lines" nos experimentos implantados entre dezembro/2003 a julho/2005. ( $\square=$ H. musciformis; $\square=$ Invertebrados; $\mathbf{\square}=\mathrm{MO}$; $=$ Outras algas).

SRPV). Observou-se uma tendência a maior percentual de MO nos tratamentos em que as algas foram inseridas em saco de nylon (SN) e em cabo de polietileno desfiado (CPD). Este recobrimento é conhecido por ocasionar perda da biomassa de espécies cultivadas em diferentes locais e esta perda é atribuída ao bloqueio da incidência de luz que interfere no crescimento da alga (Marinho-Soriano et al. 2002). A redução da taxa de crescimento de H. musciformis pela deposição de "limo" foi observada em cultivo in situ na cidade de São Sebastião, SP, por Oliveira \& Berchez (1987). Esses autores atribuíram esta deposição à menor agitação da água neste período e às variações do sentido das correntes no canal de São Sebastião.

O percentual de invertebrados sempre foi mais alto sobre as mudas cultivadas do que nas algas no costão
(Reis et al., dados em preparação). Este fato pode estar relacionado à oferta de alimento e à menor incidência de herbívoros nas unidades experimentais (Reis et al. 2005).

Observou-se não existir um padrão sazonal da taxa de crescimento de $H$. musciformis no cultivo como observados em outros estudos que variam de acordo com o local (Durako \& Dawes 1980; Berchez \& Oliveira 1990; Faccini \& Berchez 2000). Corroborando mais uma vez com a hipótese de que os fatores ambientais em microescala, ou seja, os fatores locais, são os que mais contribuem para o aumento da biomassa desta espécie (Reis \& Yoneshigue-Valentin 1998). Por exemplo, em épocas de baixa salinidade e alta precipitação, ao comparar o tratamento de H. musciformis fixada com abraçadeira (A), uma 
coletada em fevereiro com a outra coletada em abril/2005, observou-se menor taxa de crescimento em fevereiro (ANOVA, $\mathrm{F}=10,18, \mathrm{p}=0,02, \mathrm{n}=3$ ), coincidindo com a queda da biomassa no costão em fevereiro (Reis et al., dados em preparação).

Apesar dos pescadores da praia do Kutuca terem sido comunicados sobre o objetivo desse estudo, que seria uma possível nova fonte de renda em benefício deles, algumas estruturas de cultivo foram cortadas. Este fato evidencia a necessidade de conscientização da comunidade de áreas adjacentes através de projetos sociais como mencionado por Accioly (2005).

Quanto a sazonalidade fenológica, no período estudado (novembro/2003 a julho/2005), predominou a ocorrência de plantas inférteis tanto no costão (Reis et al., dados em preparação) quanto no cultivo. Em março e abril/2005, em ambos os locais, foram coletadas plantas carposporofíticas. Estes resultados diferem dos observados na Bahia, onde ocorreu maior percentual de plantas tetrasporofíticas no cultivo do que no recife. Além disso, o maior percentual de plantas femininas ocorreu no verão em ambos os locais (Wallner et al. 1992). Acredita-se que a queda na salinidade, da média de 35 para 30, pode ter sido um dos fatores responsáveis por esta resposta. Nesse estudo, a tendência a maior biomassa de carposporófitos ocorreu no outono. Por outro lado, vale ressaltar que nas duas últimas décadas, anomalias climáticas foram registradas (Goddard \& Maxx 2005), fato este que impossibilita a comparação de padrões sazonais. A ausência de exemplares masculinos de H. musciformis corresponde ao observado em estudos prévios com esta espécie nos Estados de São Paulo, da Bahia e do Rio de Janeiro (Schenkman 1989; Wallner et al. 1992; Reis \& Yoneshigue-Valentin 2000).

Não houve diferença entre os teores de carragenana das amostras coletadas no costão quando comparadas com os das mudas cultivadas (ANOVA, $\mathrm{F}=1,87, \mathrm{p}=0,22, \mathrm{n}=4$ ), que foi de $29,4 \pm 7,4 \% \mathrm{Da}$ mesma maneira, o percentual de carragenana não variou ao longo do tempo no costão (Fig. 3, ANOVA, $\mathrm{F}=2,26, \mathrm{p}=0,06, \mathrm{n}=4)$.

Concluindo, constatou-se que os ventos de quadrante norte a leste acarretaram na maior deposição de MO sobre as mudas, que desfavoreceu o crescimento desta espécie neste local. O percentual de $\mathrm{MO}$ e de Invertebrados tendeu a ser maior nas algas cultivadas do que nas do costão. Este resultado implica na necessidade de estudos sobre técnicas para evitar o acúmulo de Invertebrados nas estruturas de maricultura e da necessidade de limpeza constante das estruturas.

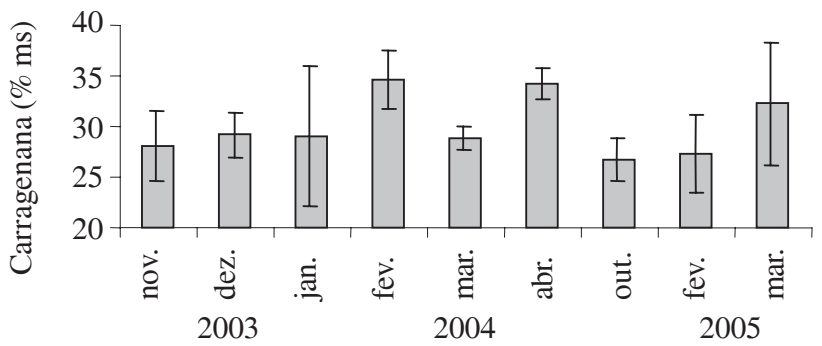

Figura 3. Percentual de carragenana de $H$. musciformis (Wulfen) J.V. Lamour. entre 2003 a 2004. Barras expressam valor médio e traços desvio padrão, $\mathrm{n}=4$.

O percentual de carragenana não diferiu entre o encontrado no costão e no cultivo, que foi em média de $30 \%$. Deste modo, acredita-se que através da análise do rendimento da carragenana de algas coletadas no local onde será implantado o cultivo pode-se ter uma estimativa do rendimento a ser obtido no cultivo. A mudança na salinidade parece ter influenciado no aparecimento de maior percentual de espécimes carposporofíticos. Acredita-se que somente com a conscientização da comunidade litorânea e da remuneração salarial de pessoas para esta atividade será possível exercer a maricultura de algas com sucesso.

\section{Agradecimentos}

À Fundação Carlos Chagas Filho de Amparo à Pesquisa do Estado do Rio de Janeiro, pelo apoio financeiro (APQ1 processo APQ1 E-26/170.191/03 e bolsa de IC processo E-26/152.303/03); ao Conselho Nacional de Desenvolvimento Científico e Tecnológico, pela bolsa PIBIC/CNPq; à Universidade Federal Rural do Rio de Janeiro e ao Centro de Adestramento da Ilha da Marambaia, pelo apoio logístico; ao Departamento de Controle do Espaço Aéreo/Serviços Regionais de Proteção ao Vôo (DECEA/SRPV) da Aeronáutica, pelo fornecimento de dados meteorológicos da Base Aérea de Santa Cruz.

\section{Referências bibliográficas}

Accioly, M.C. 2005. Esforços para estabelecer cultivos artesanais de macroalgas marinhas na Bahia. Pp. 107-114. In: Anais da X Reunião Brasileira de Ficologia. Salvador 2004. Rio de Janeiro, Museu Nacional. Série Livros 10.

Berchez, F.A.S. \& Oliveira Filho, E.C. 1990. Maricultural essays with the carragenophyte Hypnea musciformis in São Paulo, Brazil. Pp. 89-94. In: E.C.F. Oliveira \& N. Kautsky (eds.). Cultivation of seaweeds in Latin América. São Paulo, Universidade de São Paulo. 
Carvalho Filho, J. 2004. Algas uma alternativa para as comunidades costeiras? Panorama da Aquiicultura 14(84): 53 56.

Durako, M.J. \& Dawes, C.J. 1980. A comparative seasonal study of two populations of Hypnea musciformis from the East and West Coasts of Florida, USA. Marine Biology 59: 151-156.

Faccini, A.L. \& Berchez, F. 2000. Management of natural beds and standing stock evaluation of Hypnea musciformis (Gigartinales, Rhodophyta) in southeastern Brazil. Journal of Applied Phycology 12(2): 101-103.

Furtado, M.R. 1999. Alta lucratividade atrai investimentos em hidrocolóides. Química e Derivados: 21-29.

Goddard, L. \& Maxx, D. 2005. El Niño: catastrophe or opportunity. Journal of Climate 18(5): 651-665.

Marinho-Soriano, E. 2005. Cultivo experimental de Gracilaria no Rio Grande do Norte. Pp. 115-124. In: Anais da X Reunião Brasileira de Ficologia. Salvador 2004. Rio de Janeiro, Museu Nacional. Série Livros 10.

Marinho-Soriano, E.; Morales, C. \& Moreira, W.C. 2002. Cultivation of Gracilaria (Rhodophyta) in shrimp pond effluents in Brazil. Aquaculture Research 33: 1081-1086.

Miranda, G.E.C.; Bezerra, C.A.B. \& Teixeira, D.I.A. 2004. Cultivo de algas marinhas. Noções básicas. Brasília, Ed. Organização das Nações Unidades para a Agricultura e Abastecimento - FAO.

Oliveira, E.C. 1998. The seaweeds resources of Brazil. Pp. 366-371. In: A.T. Critchley \& O. Masao (eds.). Seaweeds resources of the world. Japan International Cooperation Agency.

Oliveira, E.C. \& Berchez, F.A.S. 1987. Ensayos sobre el cultivo del alga roja Hypnea musciformis (Rhodophyta, Gigartinales) en São Paulo, Brasil. Pp. 399-409. In: J.A. Verret; M. Carillo; S. Zanuy \& E.A. Huisman (eds.). Procedimientos del trabajo sobre acuicultura en America Latina. International Foundation for Science.
Paula, E.J.; Pereira, R.T.L. \& Ostini, S. 1998. Introdução de espécies exóticas de Eucheuma e Kappaphycus (Gigartinals, Rhodophyta) para fins de maricultura no litoral brasileiro: abordagem teórica e experimental. v.2. Pp. 341-357. In: Anais do IV Congresso latinoamericano, II Reunião Ibero-Americana e VII de Ficologia, Caxambu, 1996. São Paulo, Sociedade Ficológica da América Latina e Caribe.

Reis, R.P.; Barros-Barreto, M.B.; Caldeira, A.Q. \& Miranda, A.P.S. 2005. Cultivo de algas vermelha de interesse comercial na Ilha da Marambaia, Rio de Janeiro, Brasil. Pp. 287-300. In: Anais da X Reunião Brasileira de Ficologia. Salvador 2004. Rio de Janeiro, Museu Nacional. Série Livros 10.

Reis, R.P. \& Yoneshigue-Valentin, Y. 1998. Variação espaçotemporal de populações de H. musciformis (Rhodophyta - Gigartinales) na Baía de Sepetiba e Armação de Búzios, RJ, Brasil. Acta Botanica Brasilica 12(3): 465-483.

Reis, R.P. \& Yoneshigue-Valentin, Y. 2000. Phenology of H. musciformis (Wulfen) Lamouroux (Rhodophyta Gigartinales) in three populations from Rio de Janeiro state, Brazil. Botanica Marina 43: 227-230.

Romo, H.; Alveal, K. \& Werlinger, C. 2001. Growth of the commercial carrageenophyte Sarcothalia crispate (Rhodophyta, Gigartinales) on suspended culture in central Chile. Journal of Applied Phycology 13: 229-234.

Santelices, B. 1999. A concepitual framework for marine agronomy. Hydrobiologia 398/399: 15-23.

Schenkman, R.P.F. 1989. Hypnea musciformis (Rhodophyta): ecological influence on growth. Journal of Phycology 25: $192-196$.

Wallner, M.; Lobo, S.; Boccanera, N. \& Silva, E.M. 1992. Biomass, carrageenan yield and reproductive state of Hypnea musciformis (Rhodophyta : Gigartinales) under natural and experimental cultivated conditions. Aquaculture Fisheries Manage 23: 443-451.

Zar, J.H. 1996. Biostatistical analysis. Upper Saddle River, Prentice Hall. 